\title{
Hardarik Blühdorn/Sabrina Ballestracci \\ Haben italienische Deutschschreiber eine Vorliebe für hingegen? Zum Ausdruck von Adversativverknüpfungen im Sprachvergleich ${ }^{1}$
}

Der Beitrag untersucht Gemeinsamkeiten und Unterschiede in der grammatischen Gestaltung adversativer Satzverknüpfungen in der deutschen und der italienischen Schriftsprache. Die Analyse der verfügbaren Sprachmittel und ihrer Nutzung in Korpusdaten kann dazu beitragen, Auffälligkeiten in Texten italienischer Deutschschreiber zu erklären.

Schlüsselwörter: Konnektoren, adversativ, Sprachvergleich Deutsch - Italienisch

This article deals with similarities and differences in the grammatical shaping of adversative discourse relations in written German and Italian. The analysis of the available means of expression and the ways they are used in corpus data can help to explain peculiarities in German texts authored by native speakers of Italian.

Key words: connectives, adversative, comparative grammar German - Italian

\section{$1 \quad$ Einleitung}

Von Nicht-Muttersprachlern verfasste öffentliche oder halböffentliche Texte sind Kennzeichen unserer Zeit mit ihren internationalen Verflechtungen. Texte in englischer Sprache haben daran einen großen Anteil, aber auch in anderen Sprachen, etwa dem Deutschen, wird von Nicht-Muttersprachlern geschrieben und veröffentlicht. Dem aufmerksamen Leser können solche Texte, auch wenn der Autor die Fremdsprache auf sehr hohem Niveau beherrscht, vielfältige Hinweise auf dessen Muttersprache geben - ein Wissen, das auch für Sprachlehrer von Bedeutung ist, weil es Ansatzpunkte für Überlegungen zur Gestaltung des Fremdsprachenunterrichts, etwa mit dem Ziel der Vermeidung von Interferenzfehlern, liefert. So verraten sich Deutsch schreibende

\footnotetext{
Der vorliegende Beitrag präsentiert Teilergebnisse einer mehrjährigen Zusammenarbeit der Verfasser. Die Autoren haben je $50 \%$ zur Erarbeitung und Niederschrift beigetragen. Dank an Marina Foschi Albert und zwei anonyme Gutachter für wertvolle Anregungen sowie an Sandra Hansen-Morath für die statistische Begutachtung unserer Daten!
}

Muttersprachler des Russischen typischerweise in Auffälligkeiten des Artikelgebrauchs, Russisch schreibende Muttersprachler des Deutschen in Auffälligkeiten beim Verbalaspekt. Im Bereich der grammatischen Funktionswörter zeigen deutsche Englischschreiber eine merkwürdige Vorliebe für das Adverb however, italienische Deutschschreiber für das Adverb hingegen:

(1) Deutsche Geschäftsbriefe unterliegen einer strikteren Normierung in Bezug auf Sprache und Inhalt, hingegen lässt der italienische Kaufmannsstil mehr Variation zu bzw. fordert sie sogar. (Aus einem Wissenschaftstext in deutscher Sprache von einem italienischen Autor; zum Datenkorpus, auf das dieser Aufsatz sich stützt, s.u. Abschn. 4.)

Beispiel (1) ist nicht im engeren Sinne fehlerhaft, aber doch auf subtile Weise merkwürdig. Unter den Funktionswörtern des Deutschen gehört hingegen zu denjenigen, die eher selten vorkommen. Es klingt ein wenig literarisch. Ein frequenteres Synonym (vgl. Paul 1992: 159) ist dagegen, insbesondere im Vorfeld des Satzes, wo Pronominaladverbien mit $d a$-Kom- 
ponente die Verknüpfungsfunktion besser übernehmen können. ${ }^{2}$ Beispiel (1) könnte aber auch sehr gut ohne Verknüpfungsausdruck auskommen, stattdessen mit parallelem Satzbau. So wird (1a) wohl manchem Muttersprachler im Vergleich mit (1) typischer erscheinen:

(1a) Deutsche Geschäftsbriefe unterliegen einer strikteren Normierung in Bezug auf Sprache und Inhalt; der italienische Kaufmannsstil lässt mehr Variation zu bzw. fordert sie sogar.

Andererseits wird es zweifellos Muttersprachler des Deutschen geben, die (1) und (1a) als gleichermaßen unauffällig wahrnehmen und die in den hier untersuchten Texten italienischer Deutschschreiber keine Besonderheiten bemerken würden. Deshalb werden im Folgenden die zunächst nur intuitiv beobachteten Auffälligkeiten in Bezug auf hingegen genauer ins Visier genommen. Dazu werden Korpusdaten herangezogen, und es wird nach Antworten auf die folgenden Einzelfragen gesucht:

1. Wie häufig und für welche Zwecke verwenden muttersprachliche Deutschschreiber hingegen und bedeutungsverwandte Satzverknüpfer? (Abschn. 2)

2. Welche Ausdrücke werden im Italienischen für die gleichen Zwecke verwendet? (Abschn. 3)

\footnotetext{
2 Vgl. die Frequenzliste DeReWo des Instituts für Deutsche Sprache (http://www.ids-mannheim.de/derewo), die die relative Häufigkeit von mehr als 300.000 Wortformen in den IDS-Korpora (ca. 3 Mrd. Textwörter) dokumentiert. In dieser Datenbasis kommt hingegen ungefähr halb so häufig vor wie dagegen. Nicht alle Vorkommen von dagegen sind adversativ, aber nach Stichproben der Verfasser weitaus mehr als die Hälfte. Im weiteren Verlauf stützen sich die Frequenzangaben hauptsächlich auf das in Abschn. 4 vorgestellte zweisprachige Untersuchungskorpus, welches das in DeReWo dokumentierte quantitative Verhältnis zwischen hingegen und dagegen bestätigt. Ergänzend werden für hingegen, dagegen und während Belegsammlungen im Umfang von 100 bis 150 Vorkommen aus allen Bereichen der schriftlichen Kommunikation herangezogen, die über die Suchmaschine Google auf Internetseiten der Domäne .de gefunden wurden. Diese Sammlungen geben zusätzliche Aufschlüsse zum syntaktischen Verhalten und zu semantischen Verwendungsweisen der drei Konnektoren. In allen genutzten Datenquellen wurden übereinstimmende Distributionsverhältnisse gefunden.
}

3. Wie häufig und für welche Zwecke verwenden italienische Deutschschreiber hingegen und seine Synonyme? (Abschn. 4)

4. Wie lassen sich der Gebrauch, den italienische Deutschschreiber von hingegen machen, und der unidiomatische Eindruck, den er auslösen kann, erklären? (Abschn. 5)

\section{2 „Hingegen“ und andere Adversativkon- nektoren des Deutschen}

Beleg (2) aus einem Wissenschaftstext eines deutschsprachigen Autors kann als typisch für den muttersprachlichen Gebrauch von hingegen gelten:

(2) Sprechen wir über Ethik, dann suchen wir Fehler in den Moraltheorien, indem wir ihre Prinzipien auf Begriffe wie Verallgemeinerbarkeit, logische Kohärenz, Vereinbarkeit mit anderen normativen Systemen (Wissenschaft, Recht, Religion usw.) reflektieren; urteilen wir hingegen über Moral, suchen wir Fehler im konkreten Verhalten eines Menschen (im Hinblick auf eine anerkannte Moraltheorie).

Hingegen wird für textuelle Verknüpfungen mit einer ganz bestimmten semantischen, syntaktischen und informationsstrukturellen Charakteristik verwendet, die hier im Anschluss an Lohnstein (vgl. 2004: 154 ff.) als Adversativverknüpfungen bezeichnet werden. Der vorausgehende Kontext - meist der unmittelbar vorausgehende Satz - enthält einen Ausdruck A und einen Ausdruck x, die aufeinander bezogen werden. In (2) sind das die Ausdrücke sprechen über Ethik (A) und in den Moraltheorien (x). Der hingegen-Satz enthält zwei Ausdrücke B und y, die ebenfalls aufeinander bezogen werden, wobei zum einen A und $\mathrm{B}$, zum anderen $\mathrm{x}$ und $\mathrm{y}$ unter gemeinsame Oberbegriffe (,common integrators“ im Sinne von Lang 1984: 69 ff.) fallen. Der hingegen-Satz in (2) enthält die Ausdrücke urteilen über Moral (B) und im konkreten Verhalten eines Menschen (y). Sprechen über Ethik (A) und urteilen über Moral (B) fallen unter den gemeinsamen Oberbegriff „sich über Systematisierungen von Gut und Böse verständigen"; in den Moraltheorien (x) und im konkre- 
ten Verhalten eines Menschen (y) fallen unter den Oberbegriff „Kontexte für Unterscheidungen zwischen Gut und Böse". Hingegen zeigt an, dass sowohl A und B als auch $\mathrm{x}$ und $\mathrm{y}$ als Instanzen ihrer Oberbegriffe in Kontrast zueinander gesetzt sind. Abgesehen von diesem Doppelkontrast sind die Ausdrücke Ax und By - im Sinne von Pasch u. a. (vgl. 2003: 4) die Konnekte, die mit hingegen verknüpft werden - voneinander unabhängig. Anders als bei einer Konditional- oder Konzessivverknüpfung wird nicht angenommen, dass eines von beiden in irgendeiner Weise Einfluss darauf hat, ob das andere real oder wahr wird oder sonst einen Wert annimmt (vgl. Blühdorn/Golubeva 2007: 78 ff.). Die Konnekte werden zu reinen Vergleichszwecken nebeneinandergestellt.

Diese semantische Charakteristik, zusammengefasst mit der Formel „Ax vs. By“, ist die entscheidende Eigenschaft, die eine Adversativverknüpfung ausmacht und sie von anderen Verknüpfungsarten unterscheidet. Für das Deutsche ist es typisch, Adversativverknüpfungen syntaktisch als parallel gebaute Satzpaare zu gestalten. In Beispiel (2) etwa erscheinen A und B als Verberstsätze, die als Vordersätze von Konditionalverknüpfungen fungieren; $x$ und y stehen als Ortsergänzungen im Nachsatz. Der Adverbkonnektor hingegen steht immer bei By, und zwar typischerweise nicht am Satzanfang. In Beispiel (2) steht er im Mittelfeld des Konditionalsatzes.

Über die semantische und die typische syntaktische Charakteristik hinaus sind in Adversativverknüpfungen im Deutschen sehr häufig die kontrastierten Ausdrücke A, B, x und y informationsstrukturell prominent (vgl. Lohnstein 2004: 155 ff.). In mündlicher Realisierung werden sie dann akzentuiert:

(2a) Sprechen wir über ETHIK, dann suchen wir Fehler in den MORALtheorien ...; urteilen wir hingegen über MORAL, suchen wir Fehler im konkreten Verhalten eines MENSCHEN ...

Eine solche informationsstrukturelle Gestaltung unterstützt die semantischen Kontraste und geht bei parallelem Satzbau mit der Syntax Hand in Hand. Definitorisch für Adversativverknüpfungen ist aber allein die semantische Struktur. Die informationelle und vor allem die syntaktische Gestaltung kann unterschiedlich ausfallen. Hier bestehen auch Unterschiede zwischen den Gestaltungsmöglichkeiten und -konventionen in einzelnen Sprachen, etwa dem Deutschen und dem Italienischen.

Etwa doppelt so häufig wie hingegen wird im Deutschen das Adversativadverb dagegen verwendet (s. Fn. 2). Semantisch fügt es sich genau wie hingegen in die Formel „Ax vs. By“ ein. Ebenso wie hingegen steht es bevorzugt nicht am Satzanfang. Soll aber ein adversatives Adverb den Satzanfang einnehmen, so wird fast immer dagegen (oder eines der weniger frequenten Adversativadverbien wie demgegenüber) verwendet. Hingegen steht in weniger als 5\% seiner Vorkommen am Satzanfang, dagegen immerhin in $15 \%$.

Ebenso häufig wie durch Adverbien werden Adversativverknüpfungen im Deutschen durch unterordnende Konjunktionen (Subjunktoren) angezeigt, vor allem durch während. Anders als hingegen, das ausschließlich adversativ verwendet wird, hat während zunächst eine temporal-situierende Bedeutung. Adversative Lesarten kommen für etwa ein Viertel aller während-Verknüpfungen in Betracht (vgl. Blühdorn/Ravetto 2012: 59). Weitere, weniger frequente Ausdrucksmittel für Adversativverknüpfungen sind präpositional gebrauchte Wortgruppen (z.B. im Gegensatz $z u$ ) und Relationsverben (z.B. gegenüberstehen).

Besonders zu erwähnen sind neben Adversativverknüpfungen, die durch einen lexikalischen Konnektor angezeigt werden, solche wie in (1a), in denen kein Konnektor zum Einsatz kommt. Sie machen in dem untersuchten Korpus deutscher Muttersprachlertexte (s.u. Abschn. 4) ca. ein Drittel aller Adversativverknüpfungen aus und sind damit ebenso häufig wie adversative dagegen- und während-Verknüpfungen zusammen. Bei konnektorlosen Verknüpfungen gewinnen ein paralleler Satzbau und eine geeignete informationsstrukturelle Gestaltung zur Unterstützung der semantischen Kontraste an Wichtigkeit. 


\section{Adversative Satzverknüpfungen im Italienischen}

Das Italienische verfügt im Prinzip über die gleichen Ausdrucksmittel für Adversativverknüpfungen wie das Deutsche (vgl. z. B. Serianni 1991: 609 ff.). Das typischste adversativ verwendbare Adverb ist invece:

(3) È improbabile che qualcuno vi abbia mai detto che un problema filosofico è stato risolto. È invece probabile che qualcuno vi abbia fatto credere che tutti i problemi filosofici sono irrisolvibili. (,Es ist unwahrscheinlich, dass euch jemals einer berichtet hat, ein philosophisches Problem sei gelöst worden. Dagegen ist es (durchaus) wahrscheinlich, dass euch jemand hat glauben machen, alle philosophischen Probleme seien unlösbar. $)^{3}$

Beispiel (3) enthält die Ausdrücke improbabile (A) und probabile (B) sowie un problema filosofico è stato risolto (x) und tutti i problemi filosofici sono irrisolvibili (y). Zu jedem der beiden Paare gibt es einen gemeinsamen Oberbegriff („Grade von Wahrscheinlichkeit“ bzw. „Bedingungen für die Lösung philosophischer Probleme“"), unter dem es einen Gegensatz bildet. Invece kann verwendet werden, um auf die Adversativ-Konstellation „Ax vs. By" hinzuweisen. Wie hingegen, zu dem es eine semantisch nicht motivierte, rein lautliche Ähnlichkeit ${ }^{4}$ aufweist, steht es immer bei By, und zwar in der Regel (ca. 95\% der Vorkommen) nicht am Satzanfang.

Neben invece besitzt das Italienische einige weitere adversativ verwendbare Adverbien (wie diversamente) und adverbiale Präpositionalgruppen (wie al contrario), die aber insgesamt nur etwa halb so oft verwendet werden wie invece. Weniger häufig als adverbiale Adversativverknüpfungen sind solche mit einem Subjunktor, hauptsächlich mit mentre (,wäh-

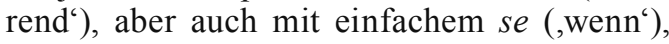

\footnotetext{
$\mathrm{Zu}$ den italienischen Belegen werden Inhaltsübersetzungen der Verfasser gegeben. Die grammatische Form kann vom Original abweichen.

4 Beide Wörter sind dreisilbig mit der Vokalfolge /I/-/e/-/ə/; beide tragen den Wortakzent auf der vorletzten Silbe; in beiden endet die Erstsilbe auf /n/, die Zweitsilbe offen. Solche zufälligen Ähnlichkeiten können Interferenzbildungen zwischen Mutter- und Fremdsprache begünstigen.
}

das gelegentlich adversativ gebraucht wird. Etwas frequenter als im Deutschen sind präpositional gebrauchte Wortgruppen wie a differenza di (,im Unterschied $\left.\mathrm{zu}^{6}\right)$. Viel seltener sind Adversativverknüpfungen ohne Konnektor:

(4) Ontologicamente il tempo è permanente, onticamente è successivo.

(,Ontologisch betrachtet ist die Zeit beständig, ontisch betrachtet vollzieht sie sich als Abfolge.')

Die Gegensatzpaare ontologicamente (A) und onticamente (B) sowie permanente (x) und successivo (y) sind gut sichtbar. Wie in Beispiel (1a) wirkt die parallele Syntax verdeutlichend. Informationsstrukturell sind die Gegensatzpaare $\mathrm{A}, \mathrm{B}, \mathrm{x}$ und $\mathrm{y}$, die außer tempo die einzigen Inhaltswörter stellen, klar prominent.

Interessanterweise scheinen Adversativverknüpfungen mit paralleler syntaktischer Gestaltung in italienischen Texten seltener $\mathrm{zu}$ sein als im Deutschen. Im deutschen Korpus haben sie einen Anteil von 50\%, im italienischen nur von $40 \%$. Nicht selten scheinen die italienischen Autoren einer parallelen syntaktischen Gestaltung der Konnekte geradezu auszuweichen, etwa in (5) (die Ausdrücke, die die Gegensatzpaare bilden, sind durch eckige Klammern angezeigt und mit Buchstabensymbolen versehen):

(5) La psicoanalisi è indagata a partire dal rapporto tra forza e senso che essa instaura, poiché in [essa] (A) [il linguaggio è capace di portare ad espressione il desiderio, che si manifesta e si nasconde nel simbolo] (x). [Il movimento dialettico] (B), invece, [individua l'origine del senso non più dietro il simbolo, ma davanti ad esso] (y) (...). (,Die Psychoanalyse wird untersucht unter dem Gesichtspunkt des Verhältnisses zwischen Kraft und Sinn, das sie postuliert, denn für sie ist die Sprache in der Lage, das Begehren zum Ausdruck zu bringen, das sich im Symbol manifestiert und zugleich versteckt. Die dialektische Bewegung dagegen erkennt den Ursprung des Sinns nicht mehr hinter dem Symbol, sondern vor ihm.')

In (6) führen zwischengeschaltete Sätze zu einer nicht-parallelen syntaktischen Gestaltung: 
(6) [Lo strutturalismo] (A) consente di liberarsi della falsa coscienza autoreferenziale e chiusa su di sé, ma rischia a sua volta di fare del linguaggio un sistema chiuso e privo di contatti con l'esterno. Esso, infatti, indaga il funzionamento di un insieme di strutture linguistiche e sociali sovraindividuali e dotate di un'intenzionalità autonoma, capaci di fornire regole generali che permettono di decifrare e classificare il comportamento umano, ma [perde così di vista il soggetto e la referenza al mondo esterno] (x).

[L'ermeneutica] (B), per Ricœur, deve invece farsi carico di un compito etico: simboli e metafore sono anche [uno strumento per la ridescrizione del mondo] (y).

(,Der Strukturalismus erlaubt es, sich von einem falschen, autoreferentiellen und in sich geschlossenen Bewusstsein zu befreien, nimmt dafür aber das Risiko in Kauf, aus der Sprache ein geschlossenes, von der Außenwelt abgeschnittenes System zu machen. Er untersucht nämlich das Zusammenspiel einer Gesamtheit von sprachlichen und überindividuellen sozialen Strukturen, die einer eigenen, ihnen innewohnenden Gesetzlichkeit folgen und allgemeine Regeln bereitstellen, die es erlauben, das menschliche Verhalten zu entschlüsseln und zu klassifizieren, verliert damit allerdings das Subjekt und die Referenz auf die Außenwelt aus dem Blick.

Für Ricœur soll sich die Hermeneutik dagegen um eine ethische Aufgabe kümmern: Symbole und Metaphern sind auch ein Werkzeug, um die Welt neu zu beschreiben.')

Eine nicht-parallele Syntax führt schnell dazu, dass die kontrastierten Ausdrücke auch informationsstrukturell nicht mehr parallel und womöglich nicht mehr prominent sind. Dann gewinnen Konnektoren und andere lexikalische Hinweise (Wörter wie Gegensatz, entgegengesetzt, unterschiedlich usw.) für die Erkennung des Adversativverhältnisses an Wichtigkeit.

Einer genaueren Untersuchung würde es möglicherweise gelingen, die Vorliebe italienischer Autoren für nicht-parallele und die deutscher Autoren für parallele Gestaltungen von Adversativverknüpfungen in einen teilerklärenden Zusammenhang mit Grammatikunterschieden zwischen beiden Sprachen zu bringen. Das kann im vorliegenden Rahmen nicht geleistet werden. Deshalb werden hier vereinfachend Stilkonventionen - die zweifellos auch eine Rolle spielen (vgl. Mortara Garavelli 2015: 188) - für die Unterschiede verantwortlich gemacht. Wichtig ist, dass beide Sprachen sehr ähnliche Ausdrucksmittel für parallele und nicht-parallele Gestaltungen von Adversativverknüpfungen zur Verfügung stellen, von denen aber ein quantitativ und qualitativ unterschiedlicher Gebrauch gemacht wird.

Die Belege (3) und (6) lassen bei genauerer Betrachtung noch eine weitere Besonderheit erkennen, die das Adverb invece betrifft und einen klaren Unterschied zu seinen deutschen Entsprechungen bildet. Hingegen ist heute ein reiner Adversativkonnektor ohne andere Bedeutungsvarianten (vgl. Paul 1992: 409). Dagegen hat außer seiner räumlichen Bedeutung ebenfalls nur die adversative (vgl. 159). Diese Ausdrücke geben damit sehr klare Hinweise auf adversative Verhältnisse. Ganz anders invece (vgl. Giacoma/Kolb 2014: 1902). In Beispiel (3) (s. o. Abschn. 2) sind zwar deutliche Gegensatzpaare erkennbar - das allein schließt aber nicht aus, dass die Konnekte in Abhängigkeit voneinander betrachtet werden und das erste als mögliche Gegenbedingung für die Wahrheit des zweiten gelten soll: Unwahrscheinlichkeit des Erstkonnekts als möglicher Hinweis auf eine analoge Unwahrscheinlichkeit des Zweitkonnekts, die dann durch invece explizit ausgeschlossen wird. In diesem Fall läge keine adversative Verknüpfung im Sinne von Lohnstein (2004: 154 ff.) vor, sondern eine konzessive im Sinne von Blühdorn/Golubeva (2007: 78 ff.). Invece wäre nicht durch ,dagegen', sondern durch ,aber ${ }^{65}$ zu übersetzen: ,Aus der Gültigkeit des ersten Konnekts darf alles geschlussfolgert werden, nur nichts, was der Gültigkeit des

\footnotetext{
5 Aber wird in Wörterbüchern und großen Teilen der Fachliteratur meist als Adversativkonnektor geführt (vgl. z. B Wöllstein u. a. 2016: 1110). Diese unglückliche terminologische Tradition beruht auf Ungenauigkeiten in der Unterscheidung zwischen adversativen und konzessiven Verhältnissen. Aber zeigt in allen Gebrauchsweisen eine Abhängigkeit zwischen seinen Konnekten an und sollte deshalb stets als Konzessivkonnektor klassifiziert werden (vgl. König 1991; Blühdorn 2017: 324). Rein entgegensetzende Adversativverknüpfungen im Sinne der hier verwendeten Definition können mit aber nicht angezeigt werden.
} 
zweiten Konnekts widersprechen würde' (vgl. auch Blühdorn/Golubeva 2007: 86 f.; Blühdorn 2008: 226). In Beispiel (6) bietet sich für invece eine Deutung als pragmatischer Marker (bzw. Diskursmarker im Sinne von Blühdorn 2017) an, der den Leser nach einem längeren Gedanken, der A mit x verbunden hat, zu der kontrastierenden Ausgangsintention des Autors zurückführen soll. Das würde nicht im Widerspruch zu seiner semantisch adversativen Funktion stehen, würde aber die pragmatische Funktion hinzufügen, auf eine gedanklich-rhetorische Umorientierung hinzuweisen.

Sowohl in konzessiver als auch in rhetorisch umorientierender Funktion (sowie in mehreren weiteren) wird invece in der Tat sehr häufig verwendet, manchmal polyfunktional wie in (6), wo die adversative durch die umorientierende Funktion ergänzt wird, manchmal mehrdeutig wie in (3), wo die adversative und die konzessive Deutung semantische Alternativen sind, zwischen denen der Interpret sich entscheiden muss - ziemlich oft auch in solcher Weise, dass adversative Deutungen ganz ausscheiden (s. u. Abschn. 4).

\section{Korpusuntersuchung}

Das Korpus, das die hauptsächliche Datengrundlage für die vorliegende Untersuchung

\begin{tabular}{|l|l|l|}
\hline Teilkorpus & Einzeltexte & laufende Textwörter \\
\hline D-Korpus & 29 & 160.863 \\
\hline ID-Korpus & 37 & 152.969 \\
\hline I-Korpus & 17 & 146.726 \\
\hline Summe & 83 & 460.558 \\
\hline
\end{tabular}

Tab. 1: Zusammensetzung des Datenkorpus

bildet, besteht aus aktuellen Wissenschaftstexten aus geisteswissenschaftlichen Fächern in deutscher und italienischer Sprache (Aufsätzen aus online publizierten Fachzeitschriften und längeren Abschnitten aus akademischen Abschlussarbeiten). Geisteswissenschaftliche Texte eignen sich besonders gut zur Untersuchung von Adversativverknüpfungen, weil sie oft Thesen und Antithesen gegenüberstellen. Das Korpus umfasst insgesamt ca. 460.000 laufende Textwörter und besteht aus drei an- nähernd gleich großen Teilkorpora ${ }^{6}$ - von Muttersprachlern verfassten Texten in deutscher (D-Korpus) und italienischer Sprache (I-Korpus) sowie von Italienern verfassten Texten auf Deutsch (ID-Korpus) (Tab. 1).

Das Korpus wurde Satz für Satz von Hand durchgearbeitet und nach der für Adversativverknüpfungen entscheidenden semantischen Konstellation „Ax vs. By“ abgesucht. Dabei wurden nur solche Stellen gezählt, an denen alle vier Kontrastausdrücke explizit realisiert waren. Implizite Kontraste, bei denen einer der Ausdrücke A, B, x oder y vom Leser inter-

\begin{tabular}{|l|l|l|l|}
\hline & D-Korpus & ID-Korpus & I-Korpus \\
\hline laufende Wörter & 160.863 & 152.969 & 146.726 \\
\hline adversative & 238 & 301 & 217 \\
Konstellationen & $1: 676$ & $1: 508$ & $1: 676$ \\
\hline ohne Konnektor & 84 & 55 & 32 \\
& $35,3 \%$ & $18,3 \%$ & $14,8 \%$ \\
\hline mit Konnektor & 154 & 246 & 185 \\
& $64,7 \%$ & $81,7 \%$ & $85,2 \%$ \\
\hline mit Adverb- & 60 & 118 & 83 \\
konnektor & $25,2 \%$ & $39,2 \%$ & $38,3 \%$ \\
\hline mit Subjunktor & 61 & 79 & 63 \\
& $25,6 \%$ & $26,2 \%$ & $29 \%$ \\
\hline mit sonstigem & 33 & 49 & 39 \\
Verknüpfer & $13,9 \%$ & $16,3 \%$ & $17,9 \%$ \\
\hline
\end{tabular}

Tab. 2: Verteilung der Adversativkonstellationen im Korpus

poliert werden muss, wurden nicht mitgezählt. Insgesamt wurden 756 Adversativkonstellationen erfasst. Tab. 2 zeigt, wie sie sich verteilen. Die Tabelle gibt zunächst allgemeine Informationen: die Zahl der Textwörter und die Gesamtzahl der Adversativkonstellationen in den Teilkorpora. In den beiden muttersprachlichen Korpora ist deren Frequenz identisch: eine Adversativkonstellation auf 676 laufende Textwörter. Im ID-Korpus ist die Frequenz mit einer Adversativkonstellation auf gut 500 Textwörter etwas höher. Das liegt an den Themen, die in diesen Texten behandelt werden: Mehrere Texte sind kontrastiv-linguistische Untersuchungen, die das Deutsche und das

\footnotetext{
Aus praktischen Gründen ist die Anzahl der Einzeltexte und damit der unterschiedlichen Autoren in den Teilkorpora etwas ungleichmäßig. Es haben sich keine Hinweise darauf ergeben, dass dies die Ergebnisse der Untersuchung beeinflusst haben könnte.
} 
Italienische gegenüberstellen. Die relative Verteilung der Sprachmittel bleibt davon unberührt.

Die dritte und vierte Tabellenzeile geben Auskunft über die Anteile von Konstellationen mit und ohne Konnektor. Sie zeigen einen deutlichen Unterschied zwischen den Texten, die von Muttersprachlern des Deutschen verfasst wurden (Spalte 1), und denjenigen, die von Muttersprachlern des Italienischen stammen (Spalten 2 und 3). Erstere formulieren mehr als ein Drittel aller Adversativkonstellationen ohne lexikalischen Konnektor, Letztere nur etwa ein Sechstel. Die für ihre Muttersprache typische Formulierungsweise behalten die italienischen Autoren in ihren deutschen Texten bei: Beim Formulieren von Adversativverknüpfungen verwenden sie erheblich mehr lexikalische Konnektoren, als es im Deutschen üblich ist.

Ein ähnliches Bild zeigt sich in der fünften Tabellenzeile, die die Verwendung adversativer Adverbkonnektoren dokumentiert. Muttersprachler des Deutschen greifen in einem Viertel der Fälle zu diesem Mittel, Muttersprachler des Italienischen zu mehr als einem Drittel, und zwar wiederum auch dann, wenn sie auf Deutsch schreiben. Die Unterschiede sind statistisch hochsignifikant. Bei den Adversativverknüpfungen mit Subjunktoren und denen mit sonstigen Verknüpfungsmitteln zeigen sich keine solchen Unterschiede. Hier ist die Verteilung in den drei Teilkorpora ähnlich. Das heißt: Dort, wo Muttersprachler des Deutschen keinen Konnektor verwenden, tendieren Muttersprachler des Italienischen dazu, einen Adverbkonnektor zu setzen.

Im I-Korpus ist invece der bevorzugte adversative Adverbkonnektor $(55$ Vorkommen $=$ gut $25 \%$ aller Adversativverknüpfungen, $66 \%$ der adversativen Adverbverknüpfungen). Im D-Korpus haben dagegen und hingegen den größten Anteil (zusammen 48 Vorkommen = $20 \%$ aller Adversativverknüpfungen, $80 \%$ der Adverbverknüpfungen). Auch im ID-Korpus dominieren dagegen und hingegen (zusammen 87 Vorkommen $=$ knapp $29 \%$ aller Adversativverknüpfungen, $74 \%$ der Adverbverknüpfungen). Beide sind deutlich häufiger als im D-Korpus, und zwar ist die Häufigkeit von hingegen stärker überhöht als die von dagegen - mit 17,6\% gegenüber $13,5 \%$ ist dagegen um knapp ein Drittel häufiger, hingegen mit $11,3 \%$ gegenüber $6,7 \%$ um mehr als zwei Drittel (Tab. 3).

\begin{tabular}{|c|c|c|c|}
\hline $\begin{array}{l}\text { adversative } \\
\text { Konstellationen }\end{array}$ & $\begin{array}{l}\text { D-Korpus } \\
238 \\
100 \% \\
\end{array}$ & \begin{tabular}{|l} 
ID-Korpus \\
301 \\
$100 \%$ \\
\end{tabular} & $\begin{array}{l}\text { I-Korpus } \\
217 \\
100 \%\end{array}$ \\
\hline $\begin{array}{l}\text { adversatives } \\
\text { invece }\end{array}$ & & & $\begin{array}{l}55 \\
25,4 \%\end{array}$ \\
\hline dagegen & $\begin{array}{l}32 \\
13,5 \% \\
\end{array}$ & $\begin{array}{l}53 \\
17,6 \% \\
\end{array}$ & \\
\hline hingegen & $\begin{array}{l}16 \\
6,7 \% \\
\end{array}$ & \begin{tabular}{|l|}
34 \\
$11,3 \%$ \\
\end{tabular} & \\
\hline Summe & $\begin{array}{l}48 \\
20 \%\end{array}$ & \begin{tabular}{|l|}
87 \\
$28,9 \%$ \\
\end{tabular} & $\begin{array}{l}55 \\
25,4 \%\end{array}$ \\
\hline
\end{tabular}

Tab. 3: Relative Häufigkeit der adversativen Adverbkonnektoren invece, dagegen und hingegen

Verknüpfungen mit dagegen bzw. hingegen sind im ID-Korpus sogar häufiger als adversative invece-Verknüpfungen im I-Korpus. Das kann erklärt werden, wenn man berücksichtigt, dass im I-Korpus zusätzlich 62 Vorkommen von invece enthalten sind, die keine adversative Deutung erlauben (s. o. Abschn. 3). Im ID-Korpus ist hingegen gelegentlich in Verknüpfungen verwendet worden, in denen es als Äquivalent für ein solches nicht-adversatives invece zu stehen scheint, etwa im folgenden Beleg:

(7) Die Regierung hat ein Mammutsparpaket angekündigt. Üblicherweise verwendet die Kanzlerin hingegen eine viel nüchternere Sprache.

Da die Kanzlerin Teil der Regierung und Ausdrücke wie Mammutsparpaket Teil der Sprache sind, kann ein Abhängigkeitsverhältnis zwischen den Konnekten hier kaum übersehen werden. Die Verknüpfung wäre somit konzessiv im Sinne von Blühdorn/Golubeva (2007: 78 ff.) zu deuten: ,Aus der Ankündigung eines Mammutsparpakets soll nicht geschlossen werden, dass die Kanzlerin sich immer so prahlerisch ausdrückt' oder: ,Dass die Kanzlerin sich üblicherweise viel nüchterner ausdrückt, konnte nicht verhindern, dass die Regierung ein Mammutsparpaket angekündigt hat.' Nach übereinstimmender Meinung der Wörterbücher und Grammatiken des Deutschen kann hingegen als Anzeiger für solche Verknüpfungen nicht korrekt verwendet wer- 
den. In (7) müsste es durch aber, allerdings oder jedoch ersetzt werden.

\section{$5 \quad$ Ergebnisse und Ausblick}

Der Aufsatz hat gezeigt, wie Intuitionen, die bei der Lektüre von nicht-muttersprachlichen Texten entstehen können, sich mit linguistischen Mitteln deskriptiv präzisieren und (mindestens teilweise) erklären lassen. Die zugrundeliegenden sprachlichen Zusammenänge haben sich im vorliegenden Fall als komplex erwiesen. Die eingangs formulierten Untersuchungsfragen können folgendermaßen beantwortet werden:

1. Hingegen ist ein ausschließlich adversativ verwendbarer Adverbkonnektor des Deutschen, der in muttersprachlichen Texten relativ selten vorkommt: im untersuchten Korpus in ca. einem Fünfzehntel aller Adversativverknüpfungen, etwa halb so häufig wie adversatives $d a$ gegen. Muttersprachler des Deutschen gestalten Adversativkonstellationen in mehr als einem Drittel der Fälle ohne lexikalischen Verknüpfer, nur durch syntaktischen und informationsstrukturellen Parallelismus. Nur in einem Viertel der Fälle wird ein Adverbkonnektor verwendet.

Der bei weitem häufigste Adversativkonnektor des Italienischen ist invece. Es wird allein für etwa ein Viertel aller Adversativkonstellationen als lexikalischer Verknüpfer verwendet. Adverbverknüpfungen insgesamt haben einen Anteil von fast $40 \%$, konnektorlose Gestaltungen einen Anteil von nur $15 \%$. Syntaktische und informationsstrukturelle Parallelgestaltung der Konnekte sind weniger typisch als im Deutschen. Invece kann als Adversativkonnektor, aber auch für mehrere nicht-adversative Verknüpfungsrelationen verwendet werden.

Italienische Deutschschreiber drücken Adversativverknüpfungen zu fast $40 \%$ durch Adverbkonnektoren aus. Konnektorlose Gestaltungen haben einen Anteil von weniger als $20 \%$. Die Verteilung ist in etwa die gleiche wie in italienischen Muttersprachlertexten. Der Anteil von hingegen ist deutlich höher als in Texten deutschsprachiger Autoren. Hingegen wird gelegentlich auch abweichend für nicht-adversative Verknüpfungen verwendet. Das kann durch die lautliche Ähnlichkeit zu invece begünstigt sein (s. Fn. 4).

2. Der Gebrauch, den italienische Deutschschreiber von hingegen machen, und seine manchmal unidiomatische Wirkung scheinen sich zum Teil aus einer lexikalischen Interferenz zu erklären: Hingegen wird fälschlich als Äquivalent für invece gebraucht. Als solches wird es in der Fachliteratur manchmal undifferenziert vorgestellt (z. B. in Schwarze 1995: 269). Wohl noch stärker wirkt sich eine Systeminterferenz aus: Italienische Deutschschreiber bevorzugen lexikalische Konnektoren, vor allem Adverbkonnektoren, wo konnektorlose Verknüpfungen typischer wären; das für das

Deutsche charakteristische Ausdrucksmittel der syntaktischen und informationsstrukturellen Parallelgestaltung wird im Vergleich zu wenig genutzt.

Für diesen letzten Punkt konnte der vorliegende Aufsatz nur eine stilistische Erklärung anführen. Diese ist höchstwahrscheinlich nicht ausreichend. Zwischen dem Deutschen und dem Italienischen bestehen tiefgreifende Unterschiede in Satzstruktur und grammatischer Kodierung der Informationsstruktur, die in diesem Aufsatz nicht dargestellt werden konnten. Dazu müssen weitere Untersuchungen folgen.

Prof. Dr. Hardarik Blühdorn

Institut für Deutsche Sprache

R5, 6-13, 68161 Mannheim

bluehdorn@ids-mannheim.de

Prof. Dr. Sabrina Ballestracci

Università degli Studi di Firenze, Dipartimento di Lingue, Letterature e Studi Interculturali

Via Santa Reparata, 93-95,

I- 50129 Florenz, Italien

sabrina.ballestracci@unifi.it 


\section{Literatur}

Blühdorn, H. (2008): Epistemische Lesarten von Satzkonnektoren - Wie sie zustande kommen und wie man sie erkennt. In: I. Pohl (Hg.), Semantik und Pragmatik - Schnittstellen. Frankfurt a.M. u. a., 217-251.

Blühdorn, H. (2017): Diskursmarker. Pragmatische Funktion und syntaktischer Status. In: H. Blühdorn u. a. (Hg.), Diskursmarker im Deutschen. Reflexionen und Analysen. Göttingen, 311-336.

Blühdorn, H./Golubeva, N. (2007): Konzessivkonnektoren und ihre morphologischen Bestandteile im Deutschen und im Russischen. In: Das Wort. Germanistisches Jahrbuch Russland 2007, 77-100.

Blühdorn, H./ Ravetto, M. (2012): Die Subjunktoren während und mentre. In: L. Cinato u. a. (Hg.), Intrecci di lingua e cultura. Studi in onore di Sandra Bosco Coletsos. Roma, 43-64.

Giacoma, L./Kolb, S. (2014): Dizionario Tedesco Italiano/Italiano - Tedesco. 3. Aufl. Bologna.

König, E. (1991): Konzessive Konjunktionen. In: A. v. Stechow/D. Wunderlich (Hg.), Semantik. Ein internationales Handbuch der zeitgenössischen Forschung. Berlin, 631-639.
Lang, E. (1984): The semantics of coordination (translation: John Pheby). Amsterdam.

Lohnstein, H. (2004): Variable und invariante Strukturmerkmale von Satzkonnektoren. In: H. Blühdorn u.a. (Hg.), Brücken schlagen. Grundlagen der Konnektorensemantik. Berlin, 137-160.

Mortara Garavelli, B. (2015): Manuale di retorica. 16. Aufl. Milano.

Pasch, R. u. a. (2003): Handbuch der deutschen Konnektoren. Linguistische Grundlagen der Beschreibung und syntaktische Merkmale der deutschen Satzverknüpfer. Berlin.

Paul, H. (1992): Deutsches Wörterbuch. 9. Aufl. von H. Henne u. a. Tübingen.

Schwarze, C. (1995): Grammatik der italienischen Sprache. 2. Aufl. Tübingen.

Serianni, L. (1991): Grammatica Italiana. Italiano comune e lingua letteraria. 2. Aufl. Bologna.

Wöllstein, A. u. a. (Hg.) (2016): Duden. Die Grammatik. 9. Aufl. Berlin (Duden, 4). 